\title{
Correction to: Cost Analysis of Robotic Roux-en-Y Gastric Bypass in a Single Academic Center: How Expensive Is Expensive?
}

\author{
Keith King ${ }^{1,2}$ (D) Alvaro Galvez ${ }^{1,2} \cdot$ Jill Stoltzfus ${ }^{1,3} \cdot$ Leonardo Claros $^{1,3} \cdot$ Maher El Chaar $^{1,3}$ \\ Published online: 20 August 2020 \\ (C) Springer Science+Business Media, LLC, part of Springer Nature 2020
}

\section{Correction to: Obesity Surgery https://doi.org/10.1007/s11695-020-04881-x}

In the original article, due to a misplaced comma and decimal point, a number that was included in Table 2, as well as in the abstract and text, was off by a factor of ten. The correct Table 2, with the correct value of $\$ 1983.62$ is shown below. Additionally, the incorrect value was copied into the abstract and text. The Results section in the abstract, with the corrected value of $\$ 1983.62$, is below:

\section{Results}

We found no difference in primary or secondary outcomes following R-RYGB and L-RYGB. The overall cost for RRYGB and L-RYGB was not statistically different (median total cost for R-RYGB and L-RYBG was $\$ 6431.34$ and $\$ 6349.09, P>0.05$, respectively). Operating time cost was significantly higher for R-RYGB compared with L-RYGB (\$2248.51 versus $\$ 1983.62$, respectively, $P<0.0001$, respectively). R-RYGB had lower cost of supplies as well as a shorter length of stay compared with L-RYGB (mean 1.5 versus 1.7 days, respectively). The same value is incorrectly entered under the Primary Outcomes section. The corrected value of $\$ 1983.62$ is shown below:

The online version of the original article can be found at https://doi.org/ 10.1007/s11695-020-04881-x

Maher El Chaar

maher.elchaar@sluhn.org

1 St. Luke's University Health Network, Bethlehem, PA, USA

2 St. Luke's University Health Network, Suite 205 North, 240 Cetronia Road, Allentown, PA 18104, USA

3 Temple Lewis Katz School of Medicine, Philadelphia, PA, USA
Overall, there were no statistically significant cost differences between R-RYGB and L-RYGB. Total median cost for RRYGB was $\$ 6431.34$ versus $\$ 6349.09$ for L-RYGB $(P>0$ .05) (Table 2). Although hospital LOS was shorter for RRYGB (mean 1.5 versus 1.7 days), the cost difference was not statistically significant (Table 2). R-RYGB operative time was longer than L-RYGB (mean 188 versus 143 mins, respectively) resulting in a significantly higher operating room time costs (median $\$ 2248.51$ versus $\$ 1983.62$, respectively, $P<0$ .0001 ) (Table 2). The cost of supplies for R-RYGB was significantly less than L-RYGB (median $\$ 3453.34$ versus $\$ 3707.58$, respectively, $P=0.007$ ) (Table 2).

One number in the Subgroup Analysis was incorrectly entered. The correct value of $\$ 6221.75$ is shown below.

Further analysis of the type of robotic platform used in RRYGB compared to L-RYGB performed during the same time period demonstrated no statistically significant cost differences between the two groups whether the da Vinci Xi platform or the da Vinci Si platform was used (Table 4). Overall cost of R-RYGB on the Xi platform was lower than the Si platform (median $\$ 6412.36$ versus $\$ 6739.96$, respectively) (Table 4).Additionally, when the cost of R-RYGB based on the type of robotic stapler was compared to the cost of L-RYGB performed during the same time period, there again was no significant difference between the groups (Table 5). Both the EndoWrist ${ }^{\circledR} 45 \mathrm{~mm}$ stapler and the SureForm ${ }^{\mathrm{TM}} 60 \mathrm{~mm}$ stapler were used with the da Vinci Xi platform. There was no significant difference between the cost of R-RYGB when the EndoWrist ${ }^{\circledR} 45 \mathrm{~mm}$ stapler or the SureForm ${ }^{\mathrm{TM}} 60 \mathrm{~mm}$ stapler was used (median $\$ 6563.82$ versus $\$ 6221.75$, respectively) (Table 5).

Publisher's Note Springer Nature remains neutral with regard to jurisdictional claims in published maps and institutional affiliations. 
Table 2 Roux-en-Y gastric bypass cost per case

\begin{tabular}{llll}
\hline & $\begin{array}{l}\text { Robotic } \\
(n=26) \\
(\text { median, range) }\end{array}$ & $\begin{array}{l}\text { Laparoscopic } \\
(n=136) \\
\text { (median, range) }\end{array}$ \\
\hline LOS & $\$ 503.23$ & $\$ 613.87$ & $(\$ 359.69-8164.35)$ \\
OR Time & $(\$ 359.69-1509.70)$ & $\$ 1983.62$ & $(\$ 1458.97-2839.54)$ \\
Supplies & $\$ 2248.51$ & $\$ 3707.58$ & .16 \\
Total Cost Per Case & $(\$ 1950.94-2779.60)$ & $(\$ 2551.73-7300.82)$ & .0001 \\
& $\$ 3453.34$ & $\$ 6349.09$ & $(\$ 5019.87-17,745.99)$ \\
\hline
\end{tabular}

*Based on separate Mann Whitney rank sums tests, chi square tests, or Fisher's exact tests as appropriate, with $\mathrm{p}<.05$ denoting statistical significance, and no adjustment for the multiple comparisons

$L O S$, Length of stay; $O R$, Operating room 